\title{
Cursos superiores de Design e Design de Informação no município do Rio de Janeiro
}

\author{
Design and Information Design courses in county of Rio de Janeiro \\ BARROS, Marina; RIBEIRO, André.
}

\begin{abstract}
RESUMO: O município do Rio de Janeiro, desde 1963, vem agregando cursos de Desenho Industrial e Design de forma pioneira. No ano de 2017 a cidade está fracionada em 160 bairros divididos em quatro zonas administrativas. Com a finalidade de descobrir as concentrações profissionais na área de Design (especialmente Design de Informação-DI) em suas diferentes modalidades de ensino superior (tecnólogos, bacharelados, licenciaturas, extensão e pós-graduações lato e stricto sensu) na cidade estabeleceu-se as perguntas: Qual a concentração espacial desses mesmos cursos? Que especialidades no ensino superior de Design a cidade dispõe? Em que áreas haveria demanda para criação de cursos de design devido à escassez de cursos em DI ou realização de eventos em DI em concentrações tradicionais de estudantes e profissionais? Onde estão as melhores oportunidades geográficas para o desenvolvimento de redes e comunidades de prática em DI? Ou mesmo se ainda vale a pena investir no segmento de ensino em Design (em geral e da Informação). Foram reunidos dados de cada instituição atuante no segmento de Design no Rio de Janeiro, seja instituição pública ou particular; presencial ou polo de educação à distância. As respostas a essa pesquisa acadêmica surpreenderão e merecem atenção de gestores de ensino, profissionais de marcado e investidores.
\end{abstract}

Palavras-chave: design de informação, mapeamento, word clouds

\begin{abstract}
The county of Rio de Janeiro, since 1963, has been adding pioneering courses in Industrial Design and Design. In the year 2017 the city is divided into 160 districts divided into four administrative zones. With the purpose of discovering the professional concentrations in the area of Design (especially Information Design-ID) in its different modalities of higher education (technologists, baccalaureate, undergraduate, extension and postgraduate studies lato and stricto sensu) in the city established the questions: What is the spatial concentration of these courses? What specialties in higher education design does the city have? In what areas would there be demand for creation of design courses due to the shortage of ID courses or ID events in traditional concentrations of students and professionals? Where are the best geographic opportunities for developing networks and communities of practice in ID? Or even if it is still worth investing in the segment of education in general Design and Information). Data were gathered from each institution working in the Design segment in Rio de Janeiro, whether public or private institution; classroom or distance education center. The answers to this academic research will surprise and merit attention from teaching managers, market professionals and investors.
\end{abstract}

Keywords: information design, mapping, word clouds

\section{Introdução}

\section{Breve História da Implantação do Ensino Superior em Design no Rio de Janeiro}

A história do ensino de Design no Brasil remete ao Rio de Janeiro. No ano de 1963, no rastro do pensamento econômico Desenvolvimentista premente no país, foi instalada no Rio de Janeiro a Escola Superior de Desenho Industrial, a ESDI, posteriormente encampada pela Universidade do Estado do Rio de Janeiro - UERJ, porém, permanecendo até os dias atuais em suas instalações originais no bairro da Lapa, região Central, como explanado no site institucional da Esdi (n.p./n.d.).

Mas essa origem carioca pode ser deslocada em até um século e meio por outros pesquisadores da história do design como Rafael Cardoso Denis em seu livro "Uma Introdução à História do Design":

Anais do $8^{\circ} \mathrm{CIDI}$ e 8 CONGIC

Guilherme Santa Rosa; Cristina Portugal (orgs.)

Sociedade Brasileira de Design da Informação - SBDI

Natal | Brasil | 2017

ISBN 978-85-212-1305-5
Proceedings of the $8^{\text {th }} \mathrm{CIDI}$ and $8^{\text {th }}$ CONGIC

Guilherme Santa Rosa; Cristina Portugal (orgs.)

Sociedade Brasileira de Design da Informação - SBDI

Natal| Brazil | 2017

ISBN 978-85-212-1305-5 
"Na época em que o ensino formal do design foi implantado no Brasil, no início da década de 1960, já datava de quase um século e meio o conflito sobre o papel da arte aplicada à indústria como agente de desenvolvimento econômico, pois o decreto assinado por D João VI em 1816 fundando a Escola Real de Ciências, Artes e Ofícios previa que a nova instituição fomentasse o "progresso da agricultura, mineralogia, indústria e comércio através do estudo das Belas Artes com aplicação e referência aos ofícios mecânicos" (DENIS, 2000:191)

João de Souza Leite relata em seu artigo "De costas para o Brasil: o ensino de um design internacionalista" algumas atividades anteriores ao estabelecimento da ESDI ocorridas na cidade de São Paulo, a respeito da implantação do ensino de Design no Brasil:

\begin{abstract}
"Um eco do III Salão de Maio, de 1939, trouxe uma revelação importante, Jacob Ruchti, o qual, junto a Lina Bo Bardi e Pietro Maria Bardi, deu início às atividades do IAC, primeira experiência de extração modernista voltada para o ensino das artes no contexto industrial. A história dessa experiência é vital para o entendimento do processo de institucionalização do ensino de design no país. O IAC, que durou de 1951 a 53, conduzido por Ruchti, propagou entre nós os laços existentes com o abstracionismo geométrico [...] Foi nessa ocasião que se estabeleceu o paradigma moderno de ensino de design no Brasil, bem como sua vinculação direta, sem intermediários, com o concretismo paulista. [...] Dessa vertente, surgiram frutos duradouros: a implantação da ESDI, no Rio de Janeiro, e da sequência de desenho industrial na Faculdade de Arquitetura e Urbanismo da USP." (LEITE, 2007:6-7)
\end{abstract}

Fato é que independente de qual ponto de partida temporal adotado para a consideração do estabelecimento formal do ensino superior de Design a cidade do Rio de Janeiro não apenas permanece em destaque como também constitui até os dias atuais o epicentro da distribuição dos demais cursos superiores nacionais que são concentrados em sua mesma região, Sudeste. Tais dados foram explicitados no artigo "A distribuição dos cursos superiores de design no Brasil":

"É importante observar a forma como estão distribuídos pelas cinco regiões: a maior concentração de cursos [de Design] está na região Sudeste e a menor [concentração de cursos de Design] na região Centro-Oeste." (SCHNAIDER; FREITAS,2016:10)

Ainda na referida pesquisa de Schnaider e Freitas é apontada a divisão atual de onze especialidades de formação em Design sendo elas: Design gráfico, design de interiores, design de moda, design de produto, web design, design digital, design de carnaval, design educacional, design de games e design publicitário. Em relação aos dados obtidos na citada pesquisa, nota-se a não existência de cursos especializados em Design de Informação em nível superior.

Sobre o parágrafo anterior, que cita as diferentes denominações de cursos e especializações da área, é relevante citar o momento atual de transição entre nomenclaturas institucionais de alguns cursos, algumas já estabelecidas e outras em processo de implantação devido a determinação emitida pelo Ministério da Educação através da SESU - Secretaria da Educação Superior quando apresentou o Referencial Nacional dos Cursos de Graduação. Esse documento propôs normatizar as nomenclaturas atuais dos cursos de Design, de maneira que substituíssem suas nomenclaturas específicas (design de moda, de mídias digitais etc.) e passassem a adotar apenas "Design" como nome do curso. As especificidades deveriam ser restritas aos cursos técnicos. Tal impacto, de fato, futuramente interferirá diretamente nos resultados desta pesquisa quando realizadas comparações entre termos. A tabela completa da transição de nomenclaturas de cursos de Design está disponível para consulta em http://portal.mec.gov.br/dmdocuments/consulta_artes.pdf

\section{Justificativa}

No ano de 2017 a cidade do Rio de Janeiro está fracionada em nada menos que 160 bairros divididos em quatro zonas administrativas. Por outro lado é conhecida a intensa expansão do ensino superior brasileiro nas últimas décadas, tanto em instituições públicas como particulares (fenômeno motivado principalmente pelos programas Reuni - Reestruturação e Expansão das Universidades Federais e Prouni - Programa Universidade para Todos; respectivamente). 
Dentro desse processo historicamente definido de contínua abertura e chegada de novas instituições de ensino assim como criação de novos cursos; é possível indagar sobre o impacto desses fenômenos recentes sobre o ensino do Design na cidade. E descobrir as concentrações profissionais na área de Design (especialmente, no caso desta pesquisa, Design de Informação-DI) em suas diferentes modalidades de ensino superior (tecnólogos, bacharelados, licenciaturas, extensão e pós-graduações lato e stricto sensu) na cidade permite elaborar algumas perguntas:

-Que especialidades no ensino superior de Design a cidade dispõe?

-Qual a concentração espacial desses mesmos cursos?

-Em que áreas haveria demanda para criação de cursos devido à escassez de oferta (seja em Design ou DI) ou realização de eventos em Design ou DI em concentrações tradicionais de estudantes e profissionais?

-Onde estão as melhores oportunidades geográficas para o desenvolvimento de redes e comunidades de prática em Design e DI?

-Ainda valeria a pena investir no segmento de ensino em Design e DI?

\section{Metodologia}

- As informações foram todas pesquisadas em site de busca de ampla aceitação (Google) nas modalidades de nível superior: graduação (tecnólogo, licenciatura e bacharelado), pós-graduação (lato e stricto sensu) e extensão.

- Sempre que possível foi pesquisado Design + "nome da universidade" e outras combinações que maximizassem os resultados da busca.

- A partir do acesso ao portal universitário, foram checados todos os links pertinentes a nível superior.

- Muitas instituições pesquisadas, embora reconhecidamente com grandes estruturas ou tradicionais, não constam na lista devido ao retorno nulo.

- Algumas instituições previamente conhecidas como ofertantes de cursos de Design também tiveram suas informações igualmente checadas através do Google e de seus respectivos portais universitários.

- Todas as informações foram organizadas no software MS Excel e posteriormente replicadas para edição em MS Word gerando uma tabela ensino $x$ bairro (TABELA 1) alocada em link específico devido a sua extensão. Os dados originais alimentaram novos gráficos, tabelas e mapas (GRÁFICO 1,2 e 3; TABELA 2, 3, 4,5 e 6; MAPAS 1, 2, 3 e 4) todos aplicados diretamente ao artigo.

\section{Mapeamento por tabela}

A opção pela análise extensiva utilizando uma tabela única segmentada em todos os 160 bairros do Rio de Janeiro se revelou profícua por evidenciar a localização (assim como as concentrações e as proximidades geográficas) entre instituições. A manutenção na listagem dos bairros sem qualquer tipo de curso em Design também permite elaborar questionamentos através dessas ausências. Entretanto, esta tabela (TABELA 1), por ser ampla demais, foi anexada separadamente para consulta posterior no link https://www.linkedin.com/pulse/tabela-detalhada-do-artigo-cursossuperiores-de-design-marina-barros Importante ressaltar que a maior parte dos dados, tópicos e outros gráficos gerados neste artigo partiram das informações organizadas na mesma.

Assim sendo, a partir dos dados extraídos da tabela 1, as principais concentrações espaciais desses cursos são atualmente a zona Central com 38 cursos, seguida pela zona Oeste com 27 cursos, zona Sul com 21 cursos e Norte com 18 cursos; 
totalizando 104 cursos de ensino superior em Design na cidade localizados em 23 bairros de um total de 160 .

GRÁFICO 1: Proporção de cursos superiores em Design $x$ Zonas

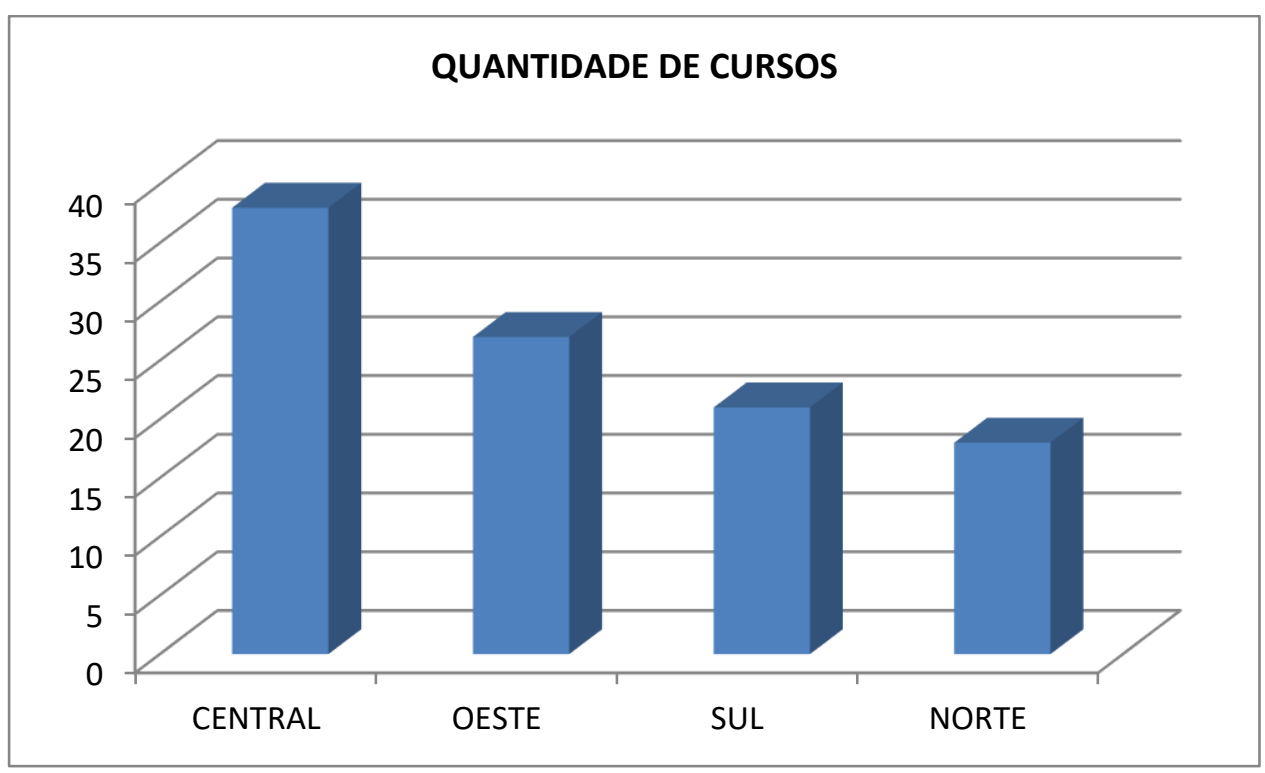

Com a finalidade de obter informações visuais a partir dos dados quantitativos apurados quanto a distribuição espacial dos 104 cursos de Design a partir da TABELA 1 , em que apenas 23 dos 160 bairros possuem cursos de design espalhados entre as quatro zonas cariocas (central, Oeste, Sul e Norte), é possível gerar uma segunda tabela, mais específica:

TABELA 2: Número de bairros o rio com cursos de design segundo suas zonas

\begin{tabular}{|c|c|c|c|}
\hline Central & Oeste & Sul & Norte \\
\hline CENTRO & BARRA DA TIJUCA & BOTAFOGO & TIJUCA \\
\hline LAPA & RECREIO DOS BAND. & COPACABANA & DEL CASTILHO \\
\hline \multirow[t]{6}{*}{ RIO COMPRIDO } & TAQUARA & FLAMENGO & PILARES \\
\hline & JARDIM SULACAP & GÁVEA & RIACHUELO \\
\hline & REALENGO & IPANEMA & BONSUCESSO \\
\hline & CAMPO GRANDE & & $\begin{array}{c}\text { CIDADE } \\
\text { UNIVERSITÁRIA }\end{array}$ \\
\hline & SANTA CRUZ & & IRAJÁ \\
\hline & & & MADUREIRA \\
\hline 3 & 7 & 5 & 8 \\
\hline \multicolumn{4}{|c|}{ Total de cursos de design segundo suas zonas } \\
\hline 28 & 27 & 21 & 18 \\
\hline
\end{tabular}

Os dados da TABELA 2 foram então explorados com os recursos do Word cloud e mapas tendo por objetivos explicitar 1- as principais instituições ofertantes de cursos de Design e 2- os eixos de bairros ofertantes de cursos de Design (em geral, dada a inexistência de cursos de Design de Informação no Brasil) dentro de cada Zona como possibilidade de exploração de redes de conhecimento, criação de comunidades de prática, percepção de grandes áreas propícias ao networking em Design, realização de eventos e desenvolvimento de novos cursos na área. 


\section{Recursos de design de informação}

\section{- Breve resumo sobre o Design da Informação e alguns recursos atuais}

A Wikipédia apresenta o Infodesign ou Design de Informação - DI como uma área do design gráfico capaz de aprimorar os meios como indivíduos adquirem informação em sistemas de comunicação. (n.p./n.d.).

A maneira como essa percepção é aperfeiçoada é através principalmente da geração de artefatos para facilitar a análise realizada pelo córtex cerebral através do sentido visual.

Retomando a pesquisa realizada com a finalidade de atender a produção deste artigo, com a identificação dos bairros com cursos de Design (independente de outros fatores quantitativos como concentração de número de cursos ou qualitativos como avaliação no Enade ou proeminência de especialidades profissionais em Design), foi sugerida a criação de eixos locais para incentivo de desenvolvimento de redes de trabalho e ensino em Design com organização de seus dados visuais segundo distribuição geográfica.

\section{- Cloud words}

A interação entre a quantidade de cursos ofertada por cada instituição dentro de cada uma das quatro Zonas espaciais do Rio foi expressa visualmente através do recurso de Cloud Words em que um peso atribuído a cada elemento na forma de palavra influenciará a forma como essa será manisfestará em termos de tamanho e intensidade de cor. Neste caso, foi utilizada a aplicação web nativa do site Worditout.

Os dados da TABELA 4 foram gerados a partir da TABELA 3 com as seguintes listas de palavras ponderadas pela quantidade de cursos em geral de cada unidade. Instituições que apresentaram mais de um campi foram somadas em um único termo de valor total.

TABELA 3: Lista de palavras ponderadas por quantidades de cursos oferecidos

\begin{tabular}{l|l|l|l} 
Central & Oeste & Sul & Norte \\
\hline Infnet:7 & Estácio:7 & Puc:13 & Estácio:6 \\
Estácio:5 & Uva:5 & Estácio:2 & Ufri:4 \\
Espm:3 & Anhanguera:4 & Facha:2 & Uva:4 \\
Esdi:13 & Unicesumar:3 & Univeritas:2 & Unicesumar:3 \\
Unicesumar:3 & Ibmr:2 & Ucam:1 & Senai-Cetiq:1 \\
Unicarioca:2 & Senac:2 & Usu:1 & \\
Anhanguera:1 & Senai-Cetiq:1 & & \\
Senac:1 & lbmec:1 & & \\
Unopar:1 & Ucb:1 & & \\
& Unopar:1 & & \\
\hline
\end{tabular}

TABELA 4: Cloud words de zonas $x$ quantitativo de cursos de cada instituição 


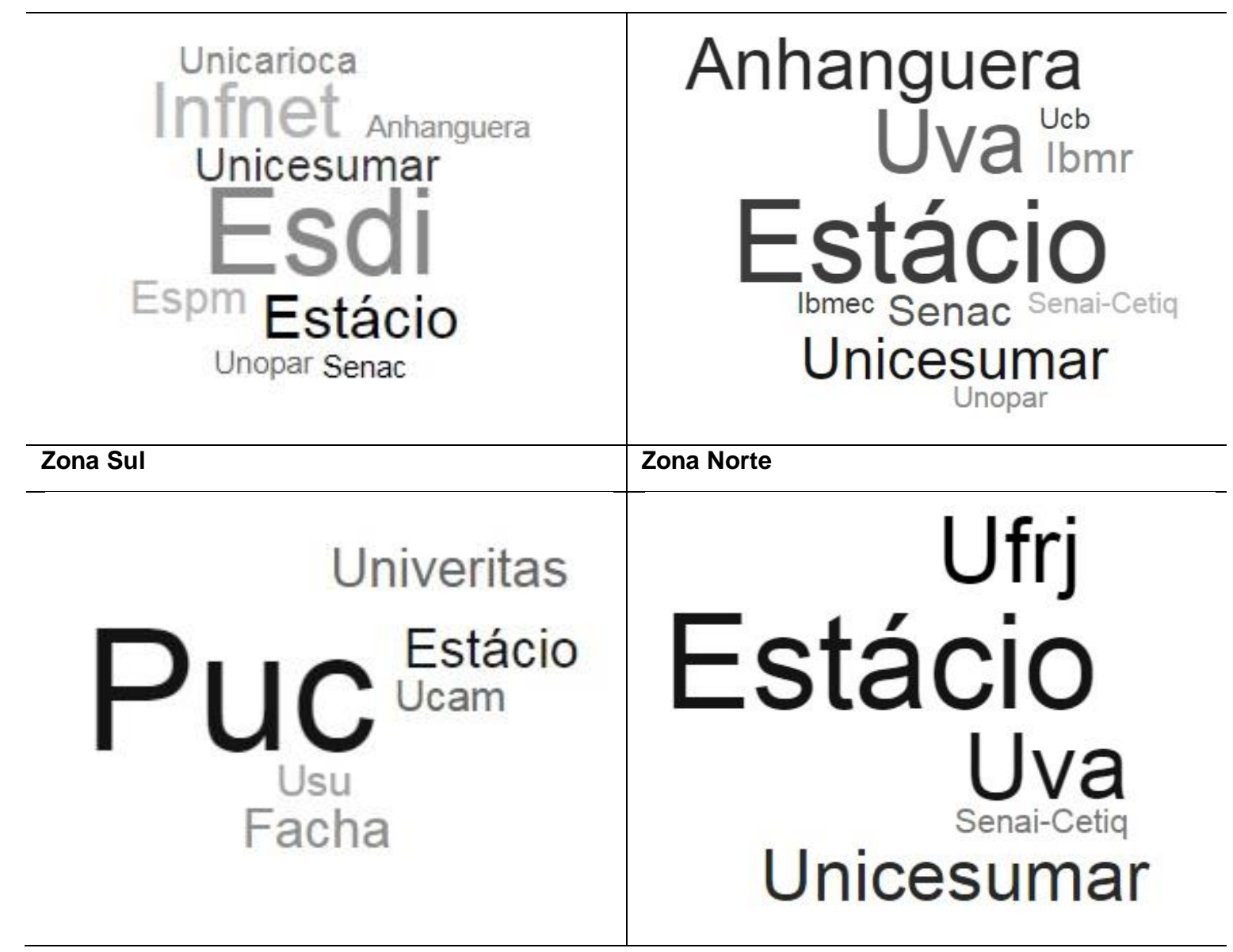

O resultado qualitativo da TABELA 3 é a observação de cada instituição de maior peso por zona geográfica segundo seu valor espacialmente contextualizado quanto ao número de cursos de Design em nível superior ofertados.

Segundo essa observação puramente quantitativa do resultado dos gráficos word clouds são, respectivamente, as instituições ofertantes de cursos de Design de maior peso em cada uma das quatro zonas: Oeste e Norte (Estácio), Central (Esdi) e Sul (Puc). Já análises do tipo qualitativa que venham a levar em consideração índices como a avaliação do Enade ou de avaliações tradicionais de mercado como o "Guia do Estudante" editado pelo Grupo Abril talvez possam gerar novos resultados.

Quando analisadas as instituições com maior número de cursos em todo o município destacase a Puc com sua concentração de 13 cursos em um único campus na Zona Sul, a Esdi Uerj que repete o mesmo com seus cursos na Lapa e a Estácio que soma 20 cursos espalhados pelo município. Também é relevante perceber que os espaços do ensino do Design no Rio de Janeiro foram francamente ocupados pelas instituições particulares quando em comparação com as unidades públicas de ensino historicamente ligadas ao pioneirismo do ensino neste campo do saber.

\section{-Mapas tradicionais}

Através do recurso Google My Maps (n.p. /n.d.) foram indicados os bairros com escolas de Design e é possível a partir de tais simulações sugerir alguns eixos visuais nas indicações fixadas no mapa. Tais eixos podem ser explorados na realização de eventos ou outras formas de convênio e associação. 


\section{\begin{tabular}{ll|l} 
CDID 2017 & $\begin{array}{l}8^{\text {th }} \mathrm{CIDI} \\
8^{\text {th }} \text { Information Design } \\
\text { International Conference }\end{array}$ & $\begin{array}{l}8^{\text {th }} \text { CONGIC } \\
8^{\text {th }} \text { Information Design } \\
\text { Student Conference }\end{array}$
\end{tabular}}

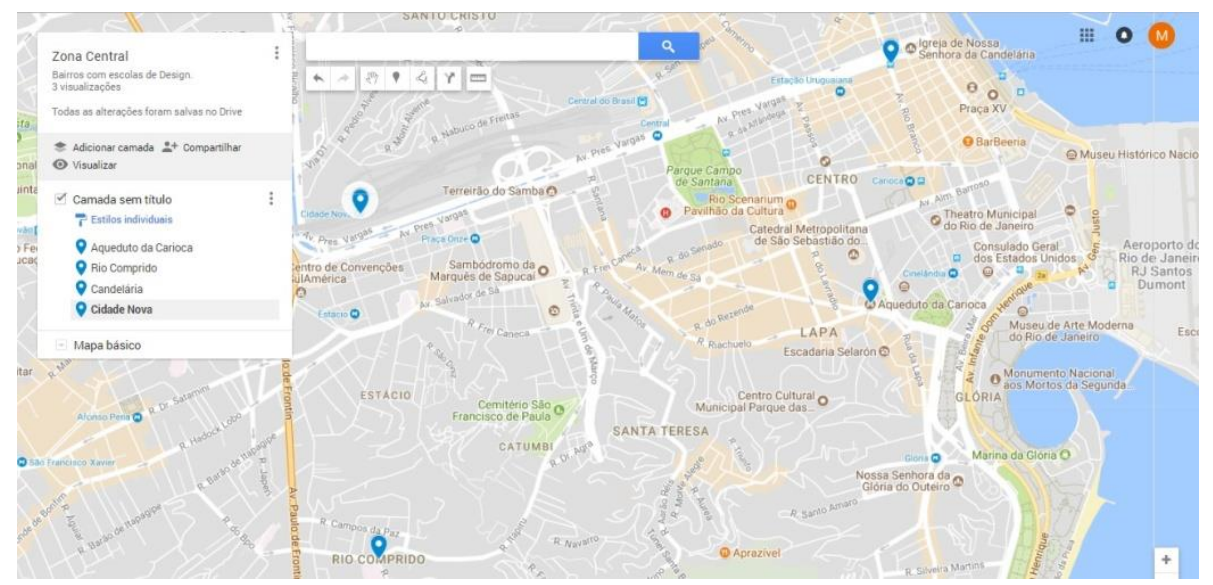

Mapa 1, sugestão de dois eixos: Centro (entre a Candelária e a Cidade Nova devido ao grande número de instituições localizadas próximas ou nesta área da cidade). Lapa x Rio Comprido.

\section{MAPA 2: Zona Sul}

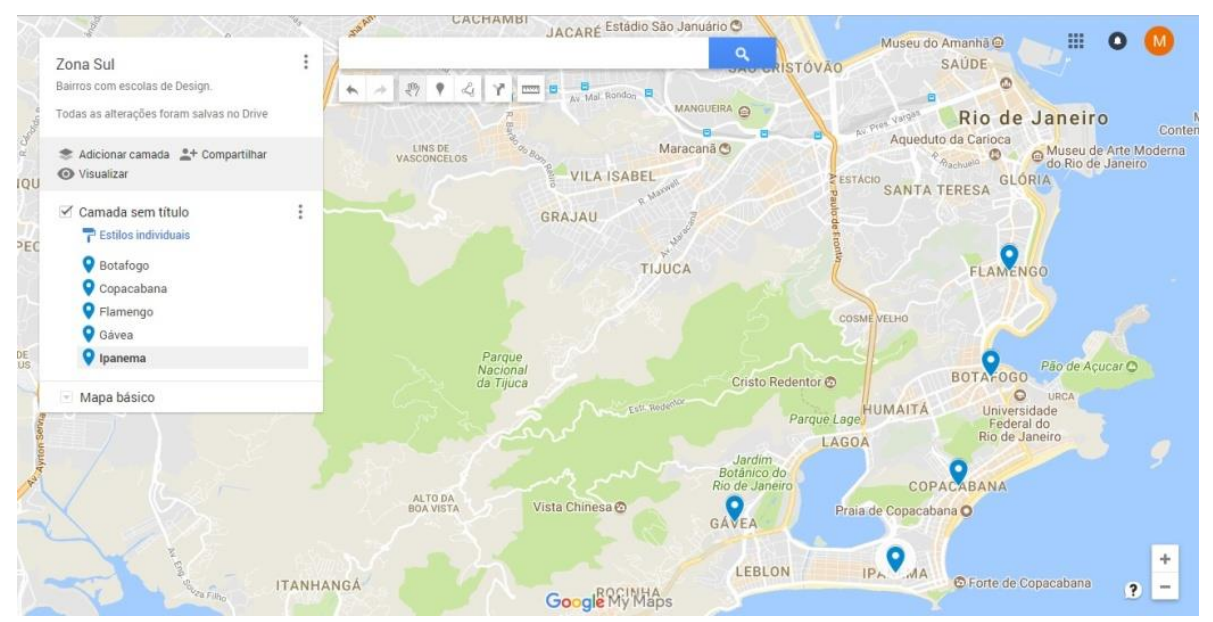

Mapa 2, sugestão de eixo único: Flamengo x Botafogo x Copacabana x Ipanema x Gávea.

\section{MAPA 3: Zona Norte}

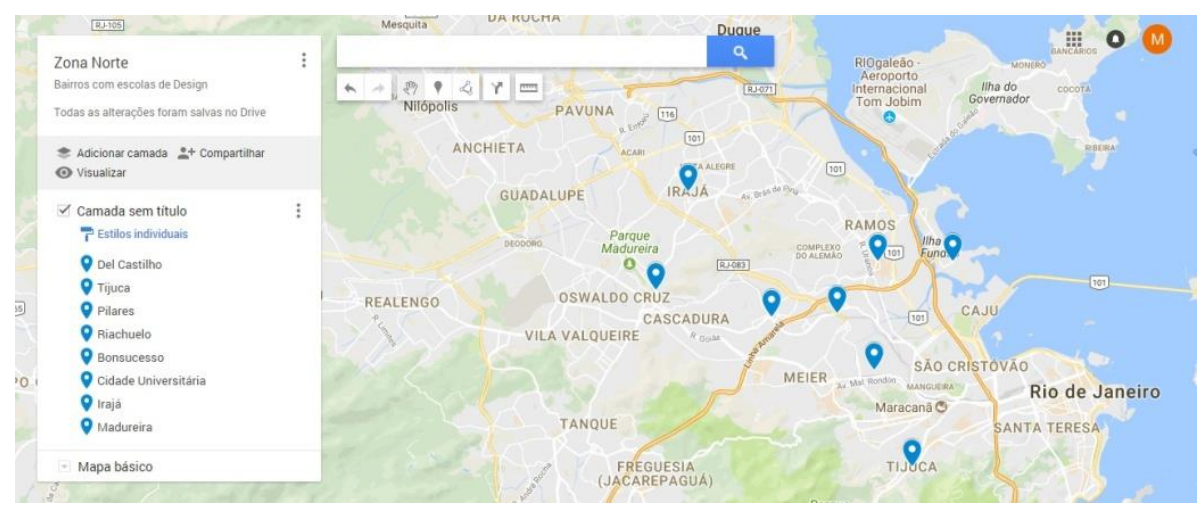

Mapa 3, sugestão de três eixos: Irajá x Madureira. Bonsucesso x Del Castilho x Cidade Universitária x Pilares. Tijuca x Riachuelo. 


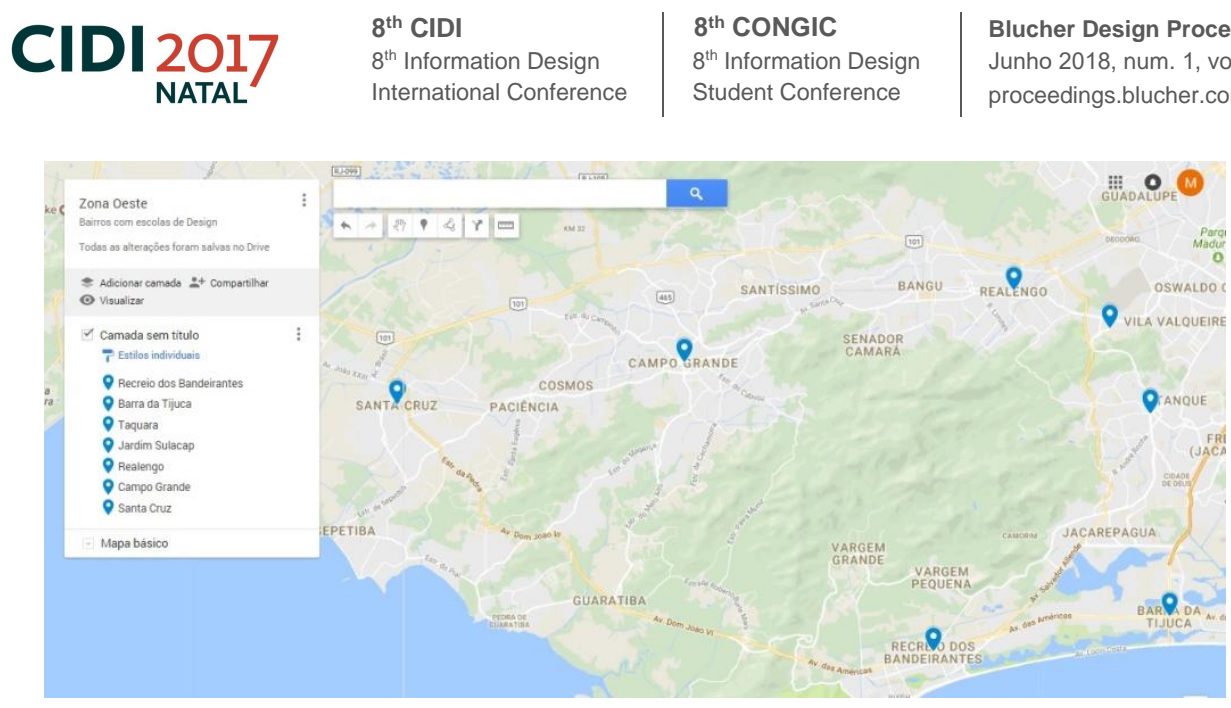

Mapa 4, sugestão de três eixos: Recreio dos Bandeirantes $x$ Barra da Tijuca. Taquara $x$ Jardim Sulacap x Realengo. Santa Cruz x Campo Grande.

\section{Análise das tabelas, mapas e word clouds}

A importância em se mapear todos os cursos de Design presentes na cidade - ao invés de apenas rapidamente constatar a não existência de cursos especialmente voltados ao Design de Informação - permitiu visualizar não apenas as características completas da oferta e demanda de cursos na cidade, mas também suas peculiaridades (concentrações espaciais e setor econômico mais atendido).

A partir da pesquisa online com a posterior categorização do mapeamento analítico (TABELA 1) foi possível realizar uma série de inferências a partir dos próprios dados obtidos e de cruzamentos diversos com informações de mercado atuais do Design e Design de Informação no município do Rio de Janeiro.

\section{1 - Respostas à justificativa desse artigo:}

- Apesar da relevante concentração de 104 cursos de Design em todo município não há cursos específicos em Design de Informação em qualquer modalidade de ensino superior.

Tal fato merece atenção devido a relevância social da atividade especializada do Design de Informação e também pelo mesmo estar comumente associado ao setor de serviços, setor econômico marcadamente ativo nesta capital.

"O Estado do Rio de Janeiro, segunda unidade da federação em termos de Produto Interno Bruto [...] setor de Serviços, [...] participa com 69\% do PIB Estadual." (CEPERJ, 2016)

- As principais concentrações espaciais desses cursos são atualmente a zona Central com 38 cursos, seguida pela zona Oeste com 27 cursos, zona Sul com 21 cursos e Norte com 18 cursos; totalizando 104 cursos de ensino superior em Design na cidade localizados em 23 bairros de um total de 160 .

Trata-se de um resultado surpreendente, porém harmonioso com o desenvolvimento histórico do Design e da própria cidade do Rio de Janeiro: o primeiro curso de Desenho Industrial foi instalado na região Central e os bairros mais populosos da capital do RJ, hoje, justamente se localizam na Zona Oeste; conforme o Painel Regional Rio de janeiro e Bairros publicado pelo Sebrae RJ.

"A cidade do Rio de Janeiro possui 6.320 .446 habitantes, o que corresponde a $40 \%$ do total do Estado do Rio de Janeiro (ERJ). A área mais populosa da capital é a Zona Norte, com 87 bairros e $42 \%$ da população do município. A maior densidade demográfica $\left(10.185 \mathrm{hab} / \mathrm{km}^{2}\right)$ também se situa na Zona Norte. A Zona Oeste apresenta a segunda maior população e a menor densidade demográfica (2.851 hab/ $\left.\mathrm{km}^{2}\right)$, já que sua área territorial é extensa. Nela estão os três bairros mais populosos da cidade: Campo Grande (328.370 hab), Bangu (244.728 hab) e Santa Cruz (217.333 
hab). A densidade demográfica do Centro e Zona Sul é de $9.794 \mathrm{hab} / \mathrm{km}^{2}$, bem próxima da Zona Norte. O bairro com a maior densidade demográfica da capital é a Rocinha (48.258 hab/km²)."

(Sebrae, 2014)

- No ano de 2017 a cidade dispõe das seguintes especialidades, em diferentes habilitações de nível superior, no ensino de Design: Design, desenho industrial, projeto de produto, programação visual, design gráfico, design de interiores, design digital, design de moda, design de animação, design de games, design de carnaval, design instrucional, comunicação visual, design e produção joalheira além de variadas especializações e extensões que podem atender a demandas específicas da formação individual.

- A partir da Tabela 1, esboçou-se a Tabela 5 geradora do Gráfico 2, comparativo quantitativamente entre Graduações x Pós-graduações no Rio de Janeiro em 2017 em suas variadas ofertas:

TABELA 5: Proporção entre Graduações x Pós-graduações no Rio de Janeiro em 2017

\begin{tabular}{l|r}
\hline Tecnólogo & 34 \\
\hline Licenciatura & 0 \\
\hline Bacharelado & 17 \\
\hline Extensão & 12 \\
\hline Total graduação & $\mathbf{6 3}$ \\
\hline Especialização & 35 \\
\hline Mestrado & 3 \\
\hline Doutorado & 3 \\
\hline Total pós-graduação & $\mathbf{4 1}$ \\
\hline
\end{tabular}

GRÁFICO 2: Proporção entre Graduações x Pós-graduações no Rio de Janeiro em 2017

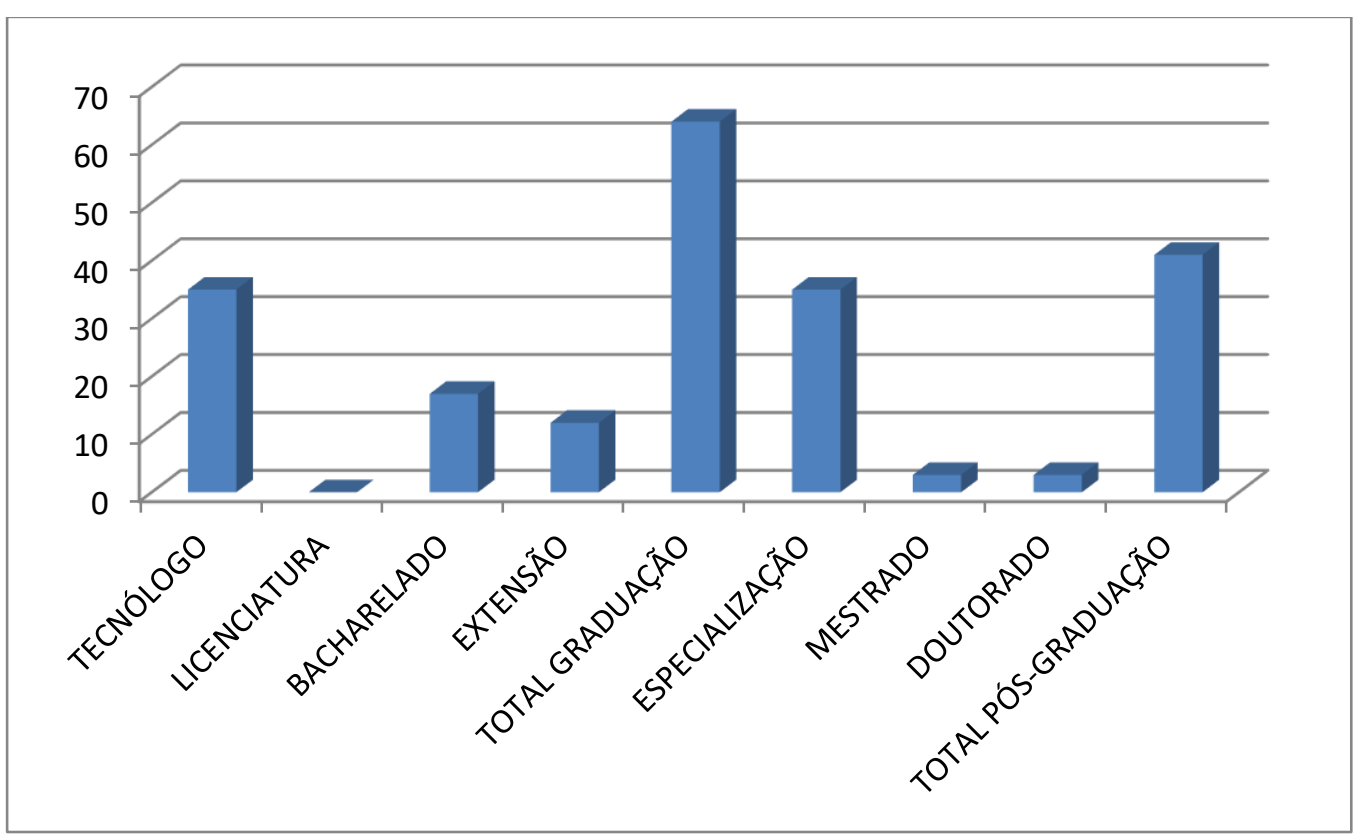

Assim, nota-se que seja razoavelmente baixa oferta de cursos de extensão, mestrado e doutorado em Design quando comparado com a oferta em todas as formas de graduação. Formam-se muitos profissionais, mas a preocupação em aperfeiçoa-los constantemente ainda se percebe incipiente, salvo a concentração explícita dos cursos de especialização. Percebe-se um mercado potencial em cursos de extensão e pós-graduação (stricto sensu) devido a característica dos mesmos em ser explorado não pela população de graduandos 
atual, mas pelo acumulado de profissionais formados por este mercado ao longo das décadas (um contingente numericamente superior e continuamente crescente até 0 momento) e também por possibilitar uma variabilidade na atualização formativa no campo do Design.

- Em uma cidade com mercado de ensino comprovadamente demandante de tais cursos em sua extensão territorial, é notável não existir cursos superiores específicos em Design de Informação, Design Antropology e Design de Superfície, atividades que vem crescendo em termos de eventos e publicações. É reduzido o número de cursos de design orientado à produção de artefatos materiais e industriais destacadamente quando em comparação com as demais especialidades do Design comumente orientadas ao setor de Serviços.

TABELA 6: Proporção entre cursos de Projeto de Produto $x$ outros cursos de Design

\begin{tabular}{l|l}
\hline $\begin{array}{l}\text { Cursos em: bacharelado ou pós em } \\
\text { design/desenho industrial e projeto de produto } \\
\text { (bacharelado ou tecnólogo) }\end{array}$ & \\
\hline Outros cursos em design & 28 \\
\hline
\end{tabular}

GRÁFICO 3: Proporção entre cursos de Projeto de Produto x outros cursos de Design

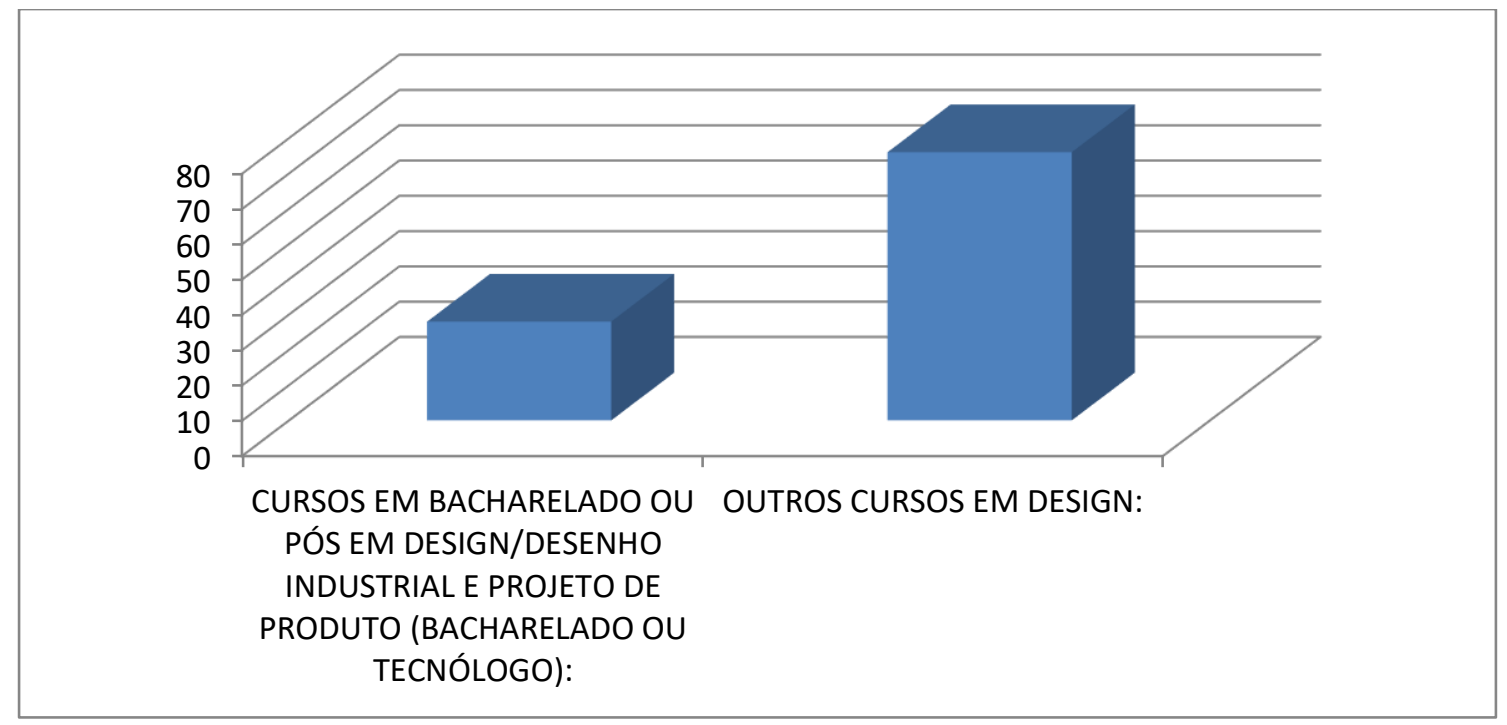

- Todas as quatro grandes zonas da cidade ofertam demanda para realização de eventos em Design assim como desenvolvimento de redes em Design, porém, são as regiões Oeste e Central as mais proeminentes em termos de número de cursos e consequente concentração e circulação de estudantes e profissionais.

Percebe-se uma correta exploração dessa característica quando verificadas a mudança nos anos recentes da Semana Design Rio e Veste Rio, que eram anteriormente realizados na Zona Sul da cidade, para a região portuária (zona Central do Rio).

- O comportamento observado no mercado quanto à questão de ainda valer a pena investir no segmento de educação superior em Design é variado, mas sugere duas tendências: investimentos na criação de cursos tecnológicos na modalidade à distância e também a opção em não investir nessa área (no caso das diversas instituições que não apresentaram nenhum tipo de curso superior orientado ao Design).

Considerando as características elementares do Design de Informação (associado tradicionalmente ao setor de serviços e a produção digital) em relação ao apurado na 
pesquisa de mapeamento analítico do ensino de Design no Rio, registra-se essa expressiva lacuna no mercado de ensino de design não apenas carioca, mas nacional.

- O resultado dos gráficos word clouds evidenciam, respectivamente, as instituições ofertante de cursos de Design de maior peso em cada uma das quatro zonas: Oeste e Norte (Estácio), Central (Infnet) e Sul (Puc).

- É relevante que os espaços do ensino do Design no Rio de Janeiro foram francamente ocupados pelas instituições particulares quando em comparação com as unidades públicas de ensino historicamente ligadas ao pioneirismo do ensino neste campo do saber.

\section{2 - Outras oportunidades observadas:}

- CCE PUC-RIO Centro não oferece nenhum curso na área de Design; o que é curioso devido ser justamente no Centro do Rio a maior concentração de escolas de Design da cidade e a mesma possuir uma ampla e tradicional estrutura de ensino de Design em sua sede na Gávea, zona Sul.

- Istituto Europeo di Design Rio e Centro Carioca de Design não oferecem cursos superiores apesar de suas localizações privilegiadas (Sul e Central, respectivamente) e relevância na área. O mesmo se repetia no Centro Design Rio, ligado ao Instituto Nacional de Tecnologia, onde as últimas notícias online são do ano de 2011.

- Destaque para as instituições privadas Unicesumar e Anhanguera que trouxeram um Tecnólogo na modalidade ead de Design de Produto, algo inédito até então.

\section{3 - Fechamento de instituições de ensino ofertantes de cursos de Design:}

- A Univercidade, Faculdade CCAA e Gama Filho, que possuíam cursos de Desenho Industrial e Design, fecharam ou faliram recentemente.

- A Faculdade Silva e Souza expandiu para mais três campi além de sua matriz, mas encerrou seu curso de Desenho Industrial, que já chegou a ser oferecido nos campus Bonsucesso e Jacarepaguá embora permaneça ofertando Arquitetura e Urbanismo.

\section{4 - Mercado de ensino associado:}

- Apesar de não contemplados nessa pesquisa, um número expressivo de universidades públicas e particulares, presenciais e à distância oferecem cursos em design na Região Metropolitana do Rio de Janeiro (Grande Rio) além de outras instituições não pesquisadas em todo o Estado.

- Apesar de não contemplados nessa pesquisa, um número expressivo de cursos privados de caráter técnicos e profissionalizantes na cidade oferecem cursos de suporte à prática do Design como ensino de desenho artístico e técnico além de uso de softwares destinados a produção de artefatos de design.

- Apesar de não contemplados nessa pesquisa, percebe-se um crescimento relevante de estúdios independentes especializados em desenvolver habilidades conceituais e artísticas de modo livre em áreas afins às demandas de conhecimento dos profissionais de Design; tais como tradicionalmente realizado há décadas pela Escola de Artes Visuais do Parque Lage: Cream-Crackers, Laje, Stampa Studio, entre outros.

\section{Conclusões}


O município do Rio de Janeiro, historicamente ligado ao desenvolvimento do ensino e prática do Design permanece engajado a esse desenvolvimento na forma da criação, expansão e permanência de cursos de Design. Percebem-se algumas possibilidades de desenvolvimento de novos cursos - como Design de Informação - capazes de elevar ainda mais o status já conquistado de uma capital com forte tradição no ensino de Design no Brasil.

A visualização da distribuição de bairros com escolas de ensino de Design em nível superior segmentados em suas quatro grandes zonas espaciais sugere a criação de eixos de desenvolvimento de ensino e prática profissional colaborando para o crescimento dessa área de conhecimento e também viabilizando novas formas de desenvolvimento econômico e profissional dos envolvidos com o Design.

Para além dos conceitos pré-concebidos a respeito de algumas instituições de grande renome no Design carioca e nacional, a visualização dos arranjos de ensino em sua localizações em toda extensão municipal permite explorar novas realidades talvez ainda não percebidas pelo mercado e agrupar indivíduos que talvez não ainda não se identifiquem enquanto membros de comunidades de prática potenciais em seus arredores.

\section{Referências}

CARDOSO, Rafael. Uma Introdução à História do Design. São Paulo: Edgar Blücher, pg 191, 2000.

LEITE, João de Souza. "De costas para o Brasil: o ensino de um design internacionalista". São Paulo: Cosac Naify, pg 6-7, 2007.

SCHNAIDER, Sílvia Helena de Carvalho; FREITAS, Sydney. A DISTRIBUIÇÃO DOS CURSOS SUPERIORES DE DESIGN NO BRASIL.. In: Anais do 2ํㅗำósio de Pós-Graduação em Design da ESDI | SPGD 2016. Anais...Rio de Janeiro(RJ) ESDI/UERJ, pg 10, 2016. https://pt.wikipedia.org/wiki/Lista_de_bairros_do_Rio_de_Janeiro_(cidade) 08/07/2017.

SITE DO CEPERJ. In: PIB. <http://www.ceperj.rj.gov.br/ceep/pib/Estimativa\%20do\%20PIB \%20do\%20Estado\%20do\%20Rio\%20de\%20Janeiro\%20em\%202016\%20revisto.doc> 20/08/2017.

SITE DA ESDI. In: História.<http://www.esdi.uerj.br/a-esdi/historia> 08/07/2017.

SITE DO GOOGLE. In: My Maps. <https://www.google.com/maps/about/mymaps/> 09/07/2017.

SITE DO MEC. In: Consulta-publica. <http://portal.mec.gov.br/dmdocuments/consulta_ artes.pdf> 27/08/2017

SITE DO SEBRAE. In: Anexos. <https://www.sebrae.com.br/Sebrae/Portal\%20Sebrae/ UFs/RJ/ Anexos/Sebrae_INFREG_2014_CapitalRJ.pdf> 08/07/2017.

SITE DO WIKIPEDIA. In: Design de Informação. <https://pt.wikipedia.org/wiki/Design_de informa\%C3\%A7\% C3\%A30> 08/07/2017.

SITE DO WORDITOUT. In: Home. <https://worditout.com/word-cloud/create> 08/07/2017.

TABELA 1. In: <https://www.linkedin.com/pulse/tabela-detalhada-do-artigo-cursos-superioresde-design-marina-barros>

\section{Sobre os autores}

Marina Vasconcelos Maluf de Barros, Licenciada, UERJ, Brazil, <marinadgrj@yahoo.com.br> André Ribeiro de Oliveira, Doutor, UERJ, Brazil <ribeiro74@gmail.com> 